TRANSACTIONS OF THE

AMERICAN MATHEMATICAL SOCIETY

Volume 349, Number 1, January 1997, Pages 271-285

S 0002-9947(97)01708-X

\title{
EVERY SEMIGROUP IS ISOMORPHIC TO A TRANSITIVE SEMIGROUP OF BINARY RELATIONS
}

\author{
RALPH MCKENZIE AND BORIS M. SCHEIN
}

\begin{abstract}
Every (finite) semigroup is isomorphic to a transitive semigroup of binary relations (on a finite set).
\end{abstract}

Let $\mathcal{B}_{A}$ be the set of all binary relations between elements of a set $A$. We consider $\mathcal{B}_{A}$ as a semigroup with the operation of relative product $\circ$. Its subsemigroups are called semigroups of binary relations. A (faithful) representation of a semigroup $\mathcal{S}$ by relations is a(n injective) homomorphism of $\mathcal{S}$ into $\mathcal{B}_{A}, A$ being any set.

A subset $\Phi \subset \mathcal{B}_{A}$ is called transitive if $\bigcup \Phi=A \times A$ (that is, for any $a, b \in A$ there exists $\varphi \in \Phi$ with $(a, b) \in \varphi)$. A representation $\mathrm{P}$ of a semigroup $\mathcal{S}$ is called transitive if $\mathrm{P}(S)$ is a transitive set of relations, that is, $\mathrm{P}$ can be viewed as a homomorphism of $\mathcal{S}$ onto a transitive semigroup of relations. A longstanding problem of semigroup theory (see [4]) asks which semigroups have faithful transitive representations by relations. An equally longstanding conjecture is: all. Various classes of semigroups (subdirectly irreducible, with zero, completely [0]-simple) were proved to have faithful transitive representations by relations (see [4] and [5]). The main results of this paper are the following theorems.

Theorem A. Every semigroup is isomorphic to a transitive semigroup of binary relations.

Theorem B. Every finite semigroup is isomorphic to a transitive semigroup of binary relations on a finite set.

Before proving the theorems, we mention some open problems.

Open Problems. 1. A relation $\varphi \subset A \times A$ is called a multipermutation if its domain and range coincide with $A$, that is, if, given any $a \in A$, there exist $b, c \in A$ with $(a, b),(c, a) \in \varphi$. Every semigroup is isomorphic to a semigroup of multipermutations [4]. Which semigroups are isomorphic to transitive semigroups of multipermutations?

2. Every set $\Phi$ of binary relations is ordered by the inclusion relation $\subset$, and every semigroup $(\Phi ; \circ)$ of relations becomes an ordered semigroup $(\Phi ; \circ ; \subset)$. Speaking of orders, we always mean partial orders. Clearly, $\subset$ is a stable order on $\Phi$ (that is, $\subset$ is a subsemigroup of the semigroup $\Phi \times \Phi$ or, equivalently,

Received by the editors September 20, 1995.

1991 Mathematics Subject Classification. Primary 20M30, 20M10; Secondary 03G15, 04A05, 05C12, 08A02, 20M12, 20M20.

Key words and phrases. Semigroup of binary relations, labeled multigraph, transitive representation.

The first author was supported in part by NSF Grant No. DMS-9596043.

(C)1997 American Mathematical Society 
$\varphi_{1} \subset \psi_{1} \wedge \varphi_{2} \subset \psi_{2} \Rightarrow \varphi_{1} \circ \psi_{1} \subset \varphi_{2} \circ \psi_{2}$ for all $\varphi_{i}, \psi_{j} \in \Phi$. Here and elsewhere $\wedge$ is a symbol of logical conjunction "and"). Every ordered semigroup $(S ; \cdot ; \leq)$ (by definition, $\leq$ is stable) is isomorphic to an ordered semigroup $(\Phi ; \circ ; \subset)$ of relations [9]; that is, there exists a bijection $R: S \rightarrow \Phi$ with $R(s t)=R(s) \circ R(t)$ and $s \leq t \Leftrightarrow R(s) \subset R(t)$ for all $s, t \in S$. We can choose $R$ preserving all existing infima of nonempty subsets of $S$ (turning them into intersections of the corresponding binary relations) [1]. Which ordered semigroups are isomorphic to transitive semigroups of binary relations ordered by set-theoretical inclusion?

Example. Let $S=\{0,1\}$ be a two-element zero semigroup ( $x y=0$ for all $x, y \in S$ ) with the order $0<1$. Then $\mathcal{S}=(S ; \cdot ; \leq)$ is an ordered semigroup. If $R$ is an isomorphism of $\mathcal{S}$ onto a transitive inclusion-ordered semigroup of relations on a set $A$, then, since $R$ is transitive and $R(0) \subset R(1)$, we obtain $R(1)=R(0) \cup R(1)=$ $A \times A$. It follows that

$$
R(0)=R\left(1^{2}\right)=R(1) \circ R(1)=(A \times A) \circ(A \times A)=A \times A=R(1),
$$

contradicting the faithfulness of $R$. Therefore, $\mathcal{S}$ is an ordered semigroup that is not isomorphic to any transitive inclusion-ordered semigroup of relations.

3. Every ordered semigroup is isomorphic to a semigroup of multipermutations ordered by inclusion [4]. Which ordered semigroups are isomorphic to transitive semigroups of multipermutations ordered by inclusion?

The remaining part of this paper is devoted to the proofs. The proof of Theorem $\mathrm{A}$ is based on constructions that may be of independent interest. This approach is discussed briefly after Theorem A is proved. For finite semigroups our constructions may yield a representation by relations on an infinite set. This is why Theorem B is given a separate proof based on a somewhat different idea.

\section{Proof of Theorem A}

Let $A$ be a set, $\mathcal{S}=(S ; \cdot)$ a semigroup, and $\mu: S \rightarrow \mathcal{B}_{A}$ a mapping. We call $\mu$ transitive if $\mu(S)$ is a transitive subset of $\mathcal{B}_{A}$. We call $\mu$ a quasi-representation if $\mu(s) \circ \mu(t) \subset \mu(s t)$ for all $s, t \in S$. Thus a representation is a quasi-representation $R$ such that $R(s) \circ R(t) \supset R(s t)$. We will extend $\mu$ to a representation $R_{\omega}$ of $\mathcal{S}$ by relations on a set $A_{\omega} \supset A$. This is done in two steps.

Step I. Here we define a quasi-representation $Q_{\mu}$. It is helpful to use the graph approach introduced now. Let $S$ and $V$ be sets called the sets of labels and vertices, respectively. A labeled multi-graph is any mapping $\mu: S \rightarrow \mathcal{B}_{V}$. A labeled arrow is a triple $(i, s, j)$ such that $(i, j) \in \mu(s)$. It is interpreted as an (oriented) arrow from $i$ to $j$ labeled by $s$, graphically $i \stackrel{s}{\rightarrow} j$. Alternatively, a labeled multigraph can be defined as just a subset of $V \times S \times V$.

A labeled path from $i$ to $j$ is a sequence of labeled arrows

$$
\pi=\left(\left(i_{0}, s_{0}, i_{1}\right),\left(i_{1}, s_{1}, i_{2}\right), \ldots,\left(i_{n-1}, s_{n-1}, i_{n}\right)\right),
$$

where $i=i_{0}$ and $j=i_{n}$. Graphically, $\pi$ is

$$
i=i_{0} \stackrel{s_{0}}{\longrightarrow} i_{1} \stackrel{s_{1}}{\longrightarrow} i_{2} \stackrel{s_{2}}{\longrightarrow} \cdots \stackrel{s_{n-2}}{\longrightarrow} i_{n-1} \stackrel{s_{n-1}}{\longrightarrow} i_{n}=j .
$$

To define the length of $\pi$ we combine the labels $s_{0}, \ldots, s_{n-1}$ in their consecutive order assuming that the set $S$ of labels is endowed with a binary operation $\cdot$, that is, $\mathcal{S}=(S ; \cdot)$ is a groupoid. The product $l(\pi)=s_{0} \cdot s_{1} \cdot \ldots \cdot s_{n-1}$ is called the length of the path $\pi$. In particular, the label $s$ of a labeled arrow $(i, s, j)$ is its length. We 
assume that - is associative, that is, $\mathcal{S}$ is a semigroup, so that $l(\pi)$ does not depend on the way in which parentheses are placed in $s_{0} \cdot s_{1} \cdot \ldots \cdot s_{n-1}$.

If $\pi_{1}: i=i_{0} \stackrel{*}{\longrightarrow} \cdots \stackrel{*}{\longrightarrow} i_{m}=j$ and $\pi_{2}: j=j_{0} \stackrel{*}{\rightarrow} \cdots \stackrel{*}{\rightarrow} j_{n}=k$ are labeled paths from $i=i_{0}$ to $j=i_{m}$ and from $j=j_{0}$ to $k=j_{n}$, respectively, their concatenation is the following labeled path $\pi_{1} \pi_{2}$ from $i$ to $k$ :

$$
\pi_{1} \pi_{2}: i=i_{0} \stackrel{*}{\rightarrow} i_{1} \cdots \stackrel{*}{\rightarrow} i_{m}=j_{0} \stackrel{*}{\rightarrow} \cdots \stackrel{*}{\rightarrow} j_{n}=k .
$$

Obviously, $l\left(\pi_{1} \pi_{2}\right)=l\left(\pi_{1}\right) l\left(\pi_{2}\right)$.

Remark on notation. A relative product $\psi \circ \varphi$ can be defined either as $(a, c) \in$ $\psi \circ \varphi \Leftrightarrow(a, b) \in \varphi \wedge(b, c) \in \psi$ for some $b$, or as $(a, c) \in \psi \circ \varphi \Leftrightarrow(a, b) \in \psi \wedge(b, c) \in \varphi$ for some $b$. If $\varphi$ and $\psi$ are mappings and $\varphi(a)=b$ stands for $(a, b) \in \varphi$, then $\psi \circ \varphi$ is a mapping such that $(\psi \circ \varphi)(a)=\psi(\varphi(a))$, and hence one should adhere to the former definition of $\circ$. This is why one of us used the former definition of $\circ$ in his previous publications. If we adhere to the former notation with the factors in a relative product written from the right to the left, and read the factors st in a product of elements of an abstract semigroup from the left to the right, we have to define a representation by the equality $\mathrm{P}(t) \circ \mathrm{P}(s)=\mathrm{P}(s t)$. This seeming contradiction to our definition can be avoided if $\varphi(a)$ is replaced by $a \varphi$ or $(a) \varphi$, in which case $(a)(\varphi \circ \psi)=((a) \varphi) \psi$. Another possibility is interpreting $\varphi(a)=b$ as $(b, a) \in \varphi$. However, this does not lie squarely with the habitual graph notation $a \stackrel{\varphi}{\longrightarrow} b$ for $\varphi(a)=b$. Thus, we may want to consider $t$ as the first factor in the product st.

Another possibility is using the latter definition of $\circ$, writing $\psi \varphi$ instead of $\psi \circ \varphi$. This notation is used in the theory of relations, but it is awkward in the semigroup context. Indeed, if $T$ and $U$ are subsets of a semigroup $\mathcal{S}$, then $T U$ ordinarily stands for the subset $\{t u \mid t \in T, u \in U\}$. If $\varphi$ and $\psi$ are relations between elements of a semigroup $\mathcal{S}$, then they are subsets of $S \times S$, which is a semigroup (the direct product of two copies of $\mathcal{S}$ ). Thus, if we want to adhere to conventional notation, we have to conclude that $\varphi \psi=\{(s u, t v) \mid(s, t) \in \varphi \wedge(u, v) \in \psi\}$. For example, if $\varphi$ is an order relation on a semigroup $\mathcal{S}$, then $\varphi \varphi \subset \varphi$ means that $\varphi$ is stable (that is, "compatible with multiplication," $s \leq t \wedge u \leq v \Rightarrow s u \leq t v$, where $a \leq b$ stands for $(a, b) \in \varphi)$. On the other hand, $\varphi \circ \varphi \subset \varphi$ means that $\varphi$ is transitive (that is, $(a, b) \in \varphi \wedge(b, c) \in \varphi \Rightarrow(a, c) \in \varphi)$.

In this paper we assume that $(a, c) \in \psi \circ \varphi \Leftrightarrow(a, b) \in \psi \wedge(b, c) \in \varphi$ for some $b$. Yet, contrary to what we have just said, we write $\mathrm{P}(s)$ instead of more logical $(s) \mathrm{P}$. Mathematics is both logical and consequential, but who said that mathematicians should be?

Let $\mathcal{S}$ be a semigroup and $\mu: S \rightarrow \mathcal{B}_{V}$ a mapping. Define a new mapping $Q_{\mu}: S \rightarrow \mathcal{B}_{V}$ as follows:

$$
Q_{\mu}(s)=\bigcup\left\{\mu\left(s_{1}\right) \circ \mu\left(s_{2}\right) \circ \cdots \circ \mu\left(s_{n}\right) \mid n \geq 1, s=s_{1} s_{2} \ldots s_{n}\right\} .
$$

Thus $(i, j) \in Q_{\mu}(s)$ when there exists a path of length $s$ leading from $i$ to $j$.

Lemma 1. $Q_{\mu}$ is a quasi-representation. If $\mu$ is transitive, so is $Q_{\mu}$.

Proof. Let $(i, k) \in Q_{\mu}(s) \circ Q_{\mu}(t)$. Then there exists $j$ such $(i, j) \in Q_{\mu}(s)$ and $(j, k) \in Q_{\mu}(t)$, that is, there exist paths $\pi_{1}$ from $i$ to $j$ and $\pi_{2}$ from $j$ to $k$ such that $l\left(\pi_{1}\right)=s$ and $l\left(\pi_{2}\right)=t$. The concatenation $\pi_{1} \pi_{2}$ is a path from $i$ to $k$ and $l\left(\pi_{1} \pi_{2}\right)=l\left(\pi_{1}\right) l\left(\pi_{2}\right)=s t$, so that $(i, k) \in Q_{\mu}(s t)$. It follows that $Q_{\mu}(s) \circ Q_{\mu}(t) \subset$ 
$Q_{\mu}(s t)$, and hence $Q_{\mu}$ is a quasi-representation of $\mathcal{S}$. Obviously, if $\mu$ is transitive, then $Q_{\mu}$ is transitive.

Step II. Now we extend quasi-representations to representations. Let $\mu: S \rightarrow$ $\mathcal{B}_{V}$ be a quasi-representation of a semigroup $\mathcal{S}$. For every $i, j \in V$ and $s, t \in S$ such that $(i, j) \in \mu(s t)$ but $(i, j) \notin \mu(s) \circ \mu(t)$, add a new vertex $k_{i, j, s, t}$ to $V$. This extends $V$ to a larger set $\bar{V}$ of vertices. For each new vertex $k_{i, j, s, t}$ add two new labeled arrows $\left(i, s, k_{i, j, s, t}\right)$ and $\left(k_{i, j, s, t}, t, j\right)$ (graphically $i \stackrel{s}{\rightarrow} k_{i, j, s, t} \stackrel{t}{\rightarrow} j$,) to the multi-graph $\mu$, thus obtaining a new multi-graph $\bar{\mu}$. Finally, extend $\bar{\mu}$ to a quasi-representation $Q_{\bar{\mu}}$ as described in Step I.

Definition. Let $Q: S \rightarrow \mathcal{B}_{A}$ and $R: S \rightarrow \mathcal{B}_{B}$ be quasi-representations of a semigroup $\mathcal{S}, A \subset B$ and $Q(s)=R(s) \cap(A \times A)$ for all $s \in S$. Then $R$ is called an extension of $Q$.

Lemma 2. $Q_{\bar{\mu}}$ is an extension of $\mu$. If $\mu$ is transitive, so is $Q_{\bar{\mu}}$.

Proof. The inclusion $\mu(r) \subset Q_{\bar{\mu}}(r) \cap(A \times A)$ is obvious. If $(a, b) \in Q_{\bar{\mu}}(r) \cap(A \times A)$, there exists a path $\pi$ from $a$ to $b$ of length $r$. The endpoints $a$ and $b$ of $\pi$ belong to the old set of vertices $A$. Therefore, new vertices can appear only in subpaths $i \stackrel{s}{\rightarrow}$ $k_{i, j, s, t} \stackrel{t}{\rightarrow} j$ of $\pi$. Replacing each of these subpaths by a labeled arrow $i \stackrel{s t}{\longrightarrow} j$, which belongs to the old multi-graph $\mu$, we obtain a new path $\pi^{\prime}$ of the same length as $\pi$ and with the same endpoints but without new vertices, so that $\pi^{\prime}$ is a path in the old multi-graph $\mu$. It follows that $(a, b) \in \mu(r)$, and hence $\mu(r)=Q_{\bar{\mu}}(r) \cap(A \times A)$.

Suppose that $\mu$ is transitive and $a, b \in \bar{A}$. If $a, b \in A$, then $(a, b) \in \mu(u)$ for some $u \in S$, and hence $(a, b) \in Q_{\bar{\mu}}(u)$. If $a \in A$ and $b \notin A$, then $b=k_{i, j, s, t}$ for some $i, j \in A$ and $s, t \in S$. Since $\mu$ is transitive, $(a, i) \in \mu(v)$ for some $v \in S$. It follows that $a \stackrel{v}{\rightarrow} i \stackrel{s}{\rightarrow} b$ is a path in $\bar{\mu}$, and hence $(a, b) \in Q_{\bar{\mu}}(v s)$. Analogously, if $a \notin A$ and $b \in A$, then $a=k_{i, j, s, t}$, also $(j, b) \in \mu(v)$ for some $v \in S$, and hence $(a, b) \in Q_{\bar{\mu}}(t) \circ Q_{\bar{\mu}}(v) \subset Q_{\bar{\mu}}(t v)$. Finally, if $a, b \notin A$, then $a=k_{i_{1}, j_{1}, s_{1}, t_{1}}$ and $b=k_{i_{2}, j_{2}, s_{2}, t_{2}}$ for some $i_{1}, i_{2}, j_{1}, j_{2} \in A$ and $s_{1}, s_{2}, t_{1}, t_{2} \in S$. Since $\mu$ is transitive, $\left(j_{1}, i_{2}\right) \in \mu(u)$ for some $u \in S$. Thus $a \stackrel{t_{1}}{\longrightarrow} j_{1} \stackrel{u}{\longrightarrow} i_{2} \stackrel{s_{2}}{\longrightarrow} b$ is a path in $\bar{\mu}$, and hence $(a, b) \in Q_{\bar{\mu}}\left(t_{1} u s_{2}\right)$. Therefore, $Q_{\bar{\mu}}$ is transitive.

If $R: S \rightarrow \mathcal{B}_{A}$ is a quasi-representation of $\mathcal{S}$, define inductively $A_{n}$ and $R_{n}$ : $S \rightarrow \mathcal{B}_{A_{n}}$ as follows. Let $A_{0}=A$ and $R_{0}=R$. If $A_{n}$ and $R_{n}: S \rightarrow \mathcal{B}_{A_{n}}$ have already been defined, let $A_{n+1}=\bar{A}_{n}$ and $R_{n+1}=Q_{\bar{R}_{n}}$. By Lemma $2, R_{n}$ is a quasi-representation of $\mathcal{S}$ by binary relations on $A_{n}$ for every $n \geq 0$.

Now define $A_{\omega}$ and $R_{\omega}$ as follows:

$$
A_{\omega}=\bigcup_{n=0}^{\infty} A_{n} \text { and } R_{\omega}(s)=\bigcup_{n=0}^{\infty} R_{n}(s) \text { for all } s \in S .
$$

Lemma 3. $R_{\omega}$ is a representation of $\mathcal{S}$ by binary relations on $A_{\omega}$ and an extension of $R_{0}$. If $R_{0}$ is transitive, so is $R_{\omega}$.

Proof. If $(i, j) \in R_{\omega}(s) \circ R_{\omega}(t)$ for some $i, j \in A_{\omega}$ and $s, t \in S$, then $(i, k) \in R_{\omega}(s)$ and $(k, j) \in R_{\omega}(t)$ for some $k \in A_{\omega}$. It follows from the definition of $R_{\omega}$ that $(i, k) \in R_{m}(s)$ and $(k, j) \in R_{n}(t)$ for some $m, n \geq 0$. If $p \geq m$ and $p \geq n$, then $R_{m}(s) \subset R_{p}(s)$ and $R_{n}(t) \subset R_{p}(t)$, so that $(i, k) \in R_{p}(s)$ and $(k, j) \in R_{p}(t)$, whence $(i, j) \in R_{p}(s) \circ R_{p}(t) \subset R_{p}(s t) \subset R_{\omega}(s t)$, because $R_{p}$ is a quasi-representation. Thus $R_{\omega}$ is a quasi-representation. 
Now let $(i, j) \in R_{\omega}(s t)$. Then $(i, j) \in R_{n}(s t)$ for some $n \geq 0$. There may exist $k \in A_{n}$ such that $(i, k) \in R_{n}(s)$ and $(k, j) \in R_{n}(t)$. If no such $k$ exists in $A_{n}$, then there exists $k=k_{i, j, s, t} \in A_{n+1}$ such that $(i, k) \in R_{n+1}(s)$ and $(k, j) \in R_{n+1}(t)$. Since $R_{\omega}$ is an extension of both $R_{n}$ and $R_{n+1}$, we obtain $(i, k) \in R_{\omega}(s)$ and $(k, j) \in R_{\omega}(t)$, and hence $(i, j) \in R_{\omega}(s) \circ R_{\omega}(t)$. Therefore, $R(s t) \subset R_{\omega}(s) \circ R_{\omega}(t)$. It follows that $R_{\omega}$ is a representation of $\mathcal{S}$. Also,

$$
R_{\omega}(s) \cap A_{m} \times A_{m}=\bigcup_{n=0}^{\infty}\left(R_{n}(s) \cap A_{m} \times A_{m}\right)=R_{m}(s),
$$

so that $R_{\omega}$ is an extension of $R_{m}$ for all $m \geq 0$.

If $R_{0}$ is transitive, then $R_{n}$ is transitive for all $n \geq 0$ by induction on $n$ and Lemma 2. If $i, j \in A_{\omega}$, then $i \in A_{m}$ and $j \in A_{n}$ for some $m$ and $n$, and hence $i, j \in A_{p}$ for every $p$ such that $p \geq m$ and $p \geq n$. Since $R_{p}$ is transitive, there exists $s \in S$ such that $(i, j) \in R_{p}(s) \subset R_{\omega}(s)$. It follows that $R_{\omega}$ is transitive.

Definition. Extend a labeled multi-graph $\mu: S \rightarrow \mathcal{B}_{A}$ to a quasi-representation $R=Q_{\mu}$ using Step I. Using Step II, extend $R$ to a representation $R_{\omega}$ of $\mathcal{S}$. We call $R_{\omega}$ a free representation of $\mathcal{S}$ generated by $\mu$.

Suppose that $\Phi$ is a transitive semigroup of relations on a set $A$ and $\Psi$ an ideal of $\Phi$ that contains a nonempty relation $\psi$ (this is so if, for example, $\Psi$ is a nonzero ideal). Let $(a, b) \in \psi$. If $i, j \in A$, then $(i, a) \in \alpha$ and $(b, j) \in \beta$ for some $\alpha, \beta \in \Phi$, because $\Phi$ is transitive. It follows that $(i, j) \in \alpha \circ \psi \circ \beta \in \Psi$, and hence $\Psi$ is transitive. Thus if a semigroup has a faithful transitive representation by relations, then every nonzero ideal of this semigroup has such a representation. A converse to this statement holds too.

Lemma 4. If an ideal of a semigroup has a faithful transitive representation by relations, then the semigroup itself has a faithful transitive representation.

Proof. Let $Q$ be a faithful transitive representation of an ideal $I$ of a semigroup $\mathcal{S}$ by relations on a set $A$. Without loss of generality, assume that $Q(s) \neq \emptyset$ for every $s \in I$. We can do that because if $Q(s)=\emptyset$ for some $s \in I$, then, since $\emptyset$ is the zero of $\mathcal{B}_{A}$ and $Q$ is faithful, $s$ is the zero of $\mathcal{S}$. In such a case replace $I$ by $\{0\}$, where $0=s$, and $Q$ by a faithful transitive representation $Q_{0}$ of $\{0\}$ by relations on the set $A_{0}=\{0\}$, where $Q_{0}(0)=\{(0,0)\}$.

Clearly, a labeled multi-graph $\mu: S \rightarrow \mathcal{B}_{A}$, defined by $\mu(s)=Q(s)$ if $s \in I$ and $\mu(s)=\emptyset$ if $s \notin I$, is a transitive quasi-representation of $\mathcal{S}$. Fix $u \in I$ and, for every $s \notin I$, choose $\left(a_{s}, b_{s}\right) \in Q(u s u)$. This is possible because $Q(u s u) \neq \emptyset$. Now add two new vertices $i_{s}$ and $j_{s}$ to $A$ and three new labeled arrows $a_{s} \stackrel{u}{\longrightarrow} i_{s} \stackrel{s}{\rightarrow} j_{s} \stackrel{u}{\rightarrow} b_{s}$ to $\mu$. Let $\hat{A}$ denote the new extended set of vertices and $\hat{\mu}$ the new labeled multi-graph with the set of vertices $\hat{A}$ and old and new labeled arrows. There is a labeled path in $\hat{\mu}$ between any two vertices of $\hat{A}$, so $Q_{\hat{\mu}}$ is a transitive quasi-representation.

Now we prove that $Q_{\hat{\mu}}(r) \cap(A \times A)$ is $Q(r)$ for $r \in I$ and $\emptyset$ for $r \notin I$. Indeed, if $(i, j) \in Q_{\hat{\mu}}(r) \cap(A \times A)$, then there is a path $\pi$ in $\hat{\mu}$ of length $r$ from $i$ to $j$. If all the vertices of $\pi$ belong to $A$, then $\pi$ is a path in $Q$, and so $(i, j) \in Q(r)$ and $r \in I$. If $\pi$ contains new vertices adjoined to $A$, then, since the endpoints of $\pi$ belong to $A$, these new vertices appear only in subpaths of the form $a_{s} \stackrel{u}{\rightarrow} i_{s} \stackrel{s}{\rightarrow} j_{s} \stackrel{u}{\rightarrow} b_{s}$ occurring in $\pi$. Replace each of these subpaths by a single labeled arrow $a_{s} \stackrel{\text { usu }}{\longrightarrow} b_{s}$ of the same length. Such arrows belong to $Q$, and in this way we replace $\pi$ by a new 
path $\pi^{\prime}$ in $Q$ with $l(\pi)=l\left(\pi^{\prime}\right)=r$. Therefore, $(i, j) \in Q(r)$ and $r \in I$. In particular, $Q_{\hat{\mu}}(r) \cap(A \times A)=\emptyset$ for $r \notin I$. It remains to notice that $Q(r) \subset Q_{\hat{\mu}}(r) \cap(A \times A)$ for all $r \in I$.

To see that $Q_{\hat{\mu}}$ is injective, let $Q_{\hat{\mu}}(s)=Q_{\hat{\mu}}(t)$. Then $Q_{\hat{\mu}}(s) \cap(A \times A)=Q_{\hat{\mu}}(t) \cap$ $(A \times A)$. Thus either $s, t \in I$, or $s, t \notin I$. In the former case, $Q(s)=Q(t)$, and hence $s=t$ because $Q$ is faithful. In the latter case, $\left(i_{s}, j_{s}\right) \in Q_{\hat{\mu}}(s)=Q_{\hat{\mu}}(t)$, and there exists a labeled path $\pi$ in $\hat{\mu}$ from $i_{s}$ to $j_{s}$ of length $t$. However, the only arrow beginning at $i_{s}$ is $i_{s} \stackrel{s}{\rightarrow} j_{s}$. If $s \neq t$, then $\pi$ must contain more than one labeled arrow. The only arrow beginning at $j_{s}$ is $j_{s} \stackrel{u}{\longrightarrow} b_{s}$. Therefore, $\pi$ begins with $i_{s} \stackrel{s}{\rightarrow} j_{s} \stackrel{u}{\rightarrow} b_{s}$. Thus $l(\pi)=\operatorname{sux}$ for some $x \in S$. Since $u \in I$, we obtain $t=l(\pi) \in I$, which is a contradiction. Thus $s=t$.

By Lemma $3, Q_{\hat{\mu}}$ extends to a transitive representation $R_{\omega}$. Also,

$$
\begin{gathered}
R_{\omega}(s)=R_{\omega}(t) \Rightarrow Q_{\hat{\mu}}(s)=R_{\omega}(s) \cap(\hat{A} \times \hat{A}) \\
=R_{\omega}(t) \cap(\hat{A} \times \hat{A})=Q_{\hat{\mu}}(t) \Rightarrow s=t,
\end{gathered}
$$

and hence $R_{\omega}$ is a faithful transitive representation of $\mathcal{S}$ by binary relations.

Definition. The kernel of a semigroup is its smallest ideal (if it exists). A semigroup $\mathcal{S}$ is called simple if it coincides with its kernel. Equivalently, $\mathcal{S}$ is simple when, given any $s, t \in S$, there exist $x, y \in S$ such that $t=x s y$.

Lemma 5. Every semigroup without a kernel possesses a faithful transitive representation.

Proof. It is a modification of the proof of Lemma 4. Let $A=\{0\}$ be a singleton set and $\mu$ a transitive representation of a semigroup $\mathcal{S}$ without a kernel by relations on $A$ defined by $\mu(s)=\{(0,0)\}$ for all $s \in S$. For every $t \in S$, choose an ideal $I_{t}$ such that $t \notin I_{t}$ and choose $u_{t} \in I_{t}$.

For every pair $(s, t)$ with $s \neq t$, add two new vertices $i_{s, t}$ and $j_{s, t}$ to $A$ and three labeled arrows $0 \stackrel{u_{t}}{\longrightarrow} i_{s, t} \stackrel{s}{\longrightarrow} j_{s, t} \stackrel{u_{t}}{\longrightarrow} 0$ to $\mu$. Let $\hat{A}$ denote the new extended set of vertices and $\hat{\mu}: S \rightarrow \mathcal{B}_{\hat{A}}$ the new labeled multi-graph. Obviously, $Q_{\hat{\mu}}$ is transitive. Extend it to a representation $R_{\omega}$ freely generated by $\hat{\mu}$. By Lemmas 2 and $3, R_{\omega}$ is transitive. If $R_{\omega}(s)=R_{\omega}(t)$ for some $s, t \in S$, then $Q_{\hat{\mu}}(s)=Q_{\hat{\mu}}(t)$. If $s \neq t$, then $\left(i_{s, t}, j_{s, t}\right) \in Q_{\hat{\mu}}(s)=Q_{\hat{\mu}}(t)$. Thus there exists a labeled path $\pi$ in $\hat{\mu}$ from $i_{s, t}$ to $j_{s, t}$ of length $t$. However, the only arrow beginning at $i_{s, t}$ is $i_{s, t} \stackrel{s}{\rightarrow} j_{s, t}$. Also, $\pi$ contains more than one labeled arrow, because $s \neq t$. The only arrow beginning at $j_{s, t}$ is $j_{s, t} \stackrel{u_{t}}{\longrightarrow} 0$. Therefore, $\pi$ begins with $i_{s, t} \stackrel{s}{\longrightarrow} j_{s, t} \stackrel{u_{t}}{\longrightarrow} 0$. Thus $l(\pi)=s u_{t} x$ for some $x \in S$. Since $u_{t} \in I_{t}$, we obtain $t=l(\pi) \in I_{t}$, contradicting $t \notin I_{t}$. Thus $s=t$ and $R_{\omega}$ is a faithful transitive representation of $S$.

To complete the proof of Theorem A, it remains to consider semigroups with kernel.

Step III. Here we define a quasi-representation $Q_{\tilde{\mu}}$ of a simple semigroup $\mathcal{S}$.

Let $E(\mathcal{S})$ be the set of all idempotents of $\mathcal{S}$ (this set may be empty). For each $e \in E(\mathcal{S})$, let $G_{e}$ denote the maximal subgroup of $\mathcal{S}$ in which $e$ is the identity element. Thus $G_{e}=\{s \in S \mid e \in s S s \wedge e s e=s\}$.

For each maximal subgroup $G_{e}$, let $\mu_{e}: G_{e} \rightarrow \mathcal{B}_{G_{e}}$ be its Cayley right regular representation. The labeled arrows of $\mu_{e}$ are $g \stackrel{h}{\rightarrow} g h$ for all $g, h \in G_{e}$. Define $\mathcal{G}(\mathcal{S})=\bigcup\left\{G_{e} \mid e \in E(\mathcal{S})\right\}$ and $\mu=\bigcup\left\{\mu_{e} \mid e \in E(\mathcal{S})\right\}$. The labeled arrows of 
$\mu: \mathcal{G}(\mathcal{S}) \rightarrow \mathcal{B}_{\mathcal{G}(\mathcal{S})}$ are $g \stackrel{h}{\rightarrow} g h$ for all $g$ and $h$ belonging to the same subgroup of $\mathcal{S}$. Extend $\mu$ to $S$ by defining $\mu(s)=\emptyset$ for all $s \notin \mathcal{G}(\mathcal{S})$.

Fix an idempotent $e_{o} \in E(\mathcal{S})$. We shall add new labeled arrows to $\mu$ connecting $G_{e_{o}}$ with $G_{e}$ in both directions, thus turning $\mu$ into a multi-graph $\tilde{\mu}$ such that $Q_{\tilde{\mu}}$ is injective on subgroups of $\mathcal{S}$.

For every $e \in E(\mathcal{S})$ there exist $r, s \in S$ such that $e_{o}=r e s$. Let $a_{e}=e_{o} r e$ and $b_{e}=e s e_{o}$. Also, assume that $a_{e_{o}}=b_{e_{o}}=e_{o}$. Then $e_{o}=a_{e} b_{e}, e_{o} a_{e}=a_{e} e=a_{e}$, and $e b_{e}=b_{e} e_{o}=b_{e}$ for every $e \in E(\mathcal{S})$. Also, $\left(b_{e} a_{e}\right)^{2}=b_{e} a_{e} b_{e} a_{e}=b_{e} e_{o} a_{e}=b_{e} a_{e}$, and hence $b_{e} a_{e} \in E(\mathcal{S})$. Let $e^{\prime}=b_{e} a_{e}$. Recall that $e \leq f \Leftrightarrow e=e f=f e$ is an order relation on $E(\mathcal{S})$. Obviously, $e^{\prime} \leq e$ for all $e \in E(\mathcal{S})$. In particular, $e_{o}^{\prime}=b_{o} a_{o}=e_{o}$.

Define $q_{e, f}=b_{e} a_{f}$ for $e, f \in E(\mathcal{S})$. Thus $e^{\prime}=q_{e, e}$.

For every $e \neq e_{o}$ add to $\mu$ two new labeled arrows $e_{o} \stackrel{a_{e}}{\longrightarrow} e$ and $e \stackrel{b_{e}}{\longrightarrow} e_{o}$. Let $\tilde{\mu}: S \rightarrow \mathcal{B}_{\mathcal{G}(\mathcal{S})}$ denote the extended labeled multi-graph. There are labeled paths in $\tilde{\mu}$ from any vertex to $e_{o}$ and from $e_{o}$ to any vertex. Combining them, we obtain paths from any vertex to any vertex. Therefore, the quasi-representation $Q_{\tilde{\mu}}$ of $\mathcal{S}$ is transitive.

Before we proceed, we prove Lemma 9, which gives exact lengths of paths in $\tilde{\mu}$. First we prove Lemmas 6-8, which are special cases of Lemma 9 and which give lengths of certain cycles, that is, paths with coinciding endpoints. Lemma 9 is stronger than what we need for completing the proof of Theorem A, but this lemma is used later in the proof of Theorem B.

Lemma 6. If $\pi: e \rightarrow \cdots \rightarrow e$ is a labeled cycle in $\tilde{\mu}$ with all vertices belonging to $G_{e}$, then all labels of $\pi$ belong to $G_{e}$ and $l(\pi)=e$.

Proof. If $i \stackrel{a}{\rightarrow} j$ is a labeled arrow in $\tilde{\mu}$ such that $i, j \in G_{e}$, then $a \in G_{e}$. Thus $\pi$ is a path in $\mu_{e}$. Since $\mu_{e}$ is the Cayley representation of $G_{e}$, we obtain $e(l(\pi))=e$, and hence $l(\pi)=e$.

Lemma 7. If $\pi: e_{o} \rightarrow \cdots \rightarrow e_{o}$ and $\tau: e \rightarrow \cdots \rightarrow e, e_{o} \neq e \in E(\mathcal{S})$, are labeled cycles in $\tilde{\mu}$ with vertices in $G_{e_{o}} \cup G_{e}$ such that some vertices of $\tau$ actually belong to $G_{e_{o}}$, then $l(\pi)=e_{o}$ and $l(\tau)=e^{\prime}$.

Proof. Obviously, $\pi$ is a concatenation of one or more cycles $\pi_{k}$ with endpoints $e_{o}$ that contain no other occurrences of $e_{o}$. It suffices to show that $l\left(\pi_{k}\right)=e_{o}$ for every $k$. If all the vertices of $\pi_{k}$ belong to $G_{e_{o}}$, then $l\left(\pi_{k}\right)=e_{o}$ by Lemma 6. Suppose that $\pi_{k}$ has vertices not belonging to $G_{e_{o}}$. The only labeled arrow leading from $G_{e_{o}}$ to $G_{e}$ is $e_{o} \stackrel{a_{e}}{\longrightarrow} e$. Having passed it, we make a (possibly trivial) cycle $\sigma$ in $G_{e}$ returning to $e$, and then pass the arrow $e \stackrel{b_{e}}{\longrightarrow} e_{o}$. By Lemma 6, $l\left(\pi_{k}\right)=a_{e} l(\sigma) b_{e}=a_{e} e b_{e}=a_{e} b_{e}=e_{o}$.

Also, $\tau$ is a concatenation of a cycle $\tau_{1}$ in $G_{e}$, an arrow $e \stackrel{b_{e}}{\longrightarrow} e_{o}$, a cycle $\tau_{2}: e_{o} \rightarrow$ $\cdots \rightarrow e_{o}$, an arrow $e_{o} \stackrel{a_{e}}{\longrightarrow} e$, and a cycle $\tau_{3}$ in $G_{e}$. By Lemma $6, l\left(\tau_{1}\right)=e=l\left(\tau_{3}\right)$. By the first part of Lemma 7, $l\left(\tau_{2}\right)=e_{o}$. Thus $l(\pi)=e b_{e} e_{o} a_{e} e=e e^{\prime} e=e^{\prime}$.

Lemma 8. Let $\pi: e \rightarrow \cdots \rightarrow e$ be a labeled cycle in $\tilde{\mu}$ with vertices in $\mathcal{G}(\mathcal{S})$. If all vertices of $\pi$ are in $G_{e}$, then $l(\pi)=e$. Otherwise, $l(\pi)=e^{\prime}$.

Proof. If all vertices of $\pi$ are in $G_{e}$, apply Lemma 6 . Let $\pi$ have vertices not belonging to $G_{e}$. If $e=e_{o}$, then $\pi$ is a concatenation of cycles $\pi_{k}$ with endpoints $e_{o}$, where each of $\pi_{k}$ has no other occurrences of $e_{o}$. If $e_{o}, e_{1}$, and $e_{2}$ are three different idempotents, then any path from $e_{1}$ to $e_{2}$ passes through $e_{o}$. Therefore, either $\pi_{k}$ 
is a path with all vertices in $G_{e_{o}}$, or it is a path with vertices in $G_{e_{o}} \cup G_{e}$ for some $e \in E(\mathcal{S})$. By Lemma 7, $l\left(\pi_{k}\right)=e_{o}$. Thus $l(\pi)=l\left(\pi_{1}\right) l\left(\pi_{2}\right) \ldots=e_{o} e_{o} \ldots=e_{o}$.

Now let $e \neq e_{o}$. If $\pi$ has no occurrences of $e$, except the endpoints, then it is of the form $e \stackrel{b_{e}}{\longrightarrow} e_{o} \rightarrow \cdots \rightarrow e_{o} \stackrel{a_{e}}{\longrightarrow} e$. As we have just seen, the length of $e_{o} \rightarrow \cdots \rightarrow e_{o}$ is $e_{o}$, and hence $l(\pi)=b_{e} e_{o} a_{e}=b_{e} a_{e}=e^{\prime}$. In general, $\pi$ is a concatenation of cycles $\pi_{k}$ with endpoints $e$, where $\pi_{k}$ contain no other occurrences of $e$. Then $l\left(\pi_{k}\right)$ is $e$ or $e^{\prime}$, and $l(\pi)$ is a product of idempotents $e$ and $e^{\prime}$. Since $e e^{\prime}=e^{\prime} e=e^{\prime}$, we obtain $l(\pi)=e^{\prime}$.

Lemma 9. Let $\pi$ be a labeled path from $i$ to $j$ in $\tilde{\mu}$, where $i \in G_{e}$ and $j \in G_{f}$ for certain idempotents $e$ and $f$ of a simple semigroup $\mathcal{S}$. If all vertices of $\pi$ belong to $G_{e}$, then $l(\pi)=i^{-1} j$. Otherwise, $l(\pi)=i^{-1} q_{e, f} j$.

Proof. If all the vertices of $\pi$ are in $G_{e}$, then $\pi$ is a path in $\mu_{e}$, and hence $i(l(\pi))=j$. Thus $l(\pi) \in G_{e}$ and $l(\pi)=i^{-1} j$. If some vertices of $\pi$ are not in $G_{e}$, then $\pi$ is a concatenation of paths $\pi_{1}$ from $i$ to $e, \sigma$ from $e$ to $f$, and $\pi_{2}$ from $f$ to $j$, where all the vertices of $\pi_{1}$ are in $G_{e}$ and all those of $\pi_{2}$ in $G_{f}$. Since $\pi_{1}$ and $\pi_{2}$ are paths in $\mu_{e}$ and $\mu_{f}$, respectively, we obtain $l\left(\pi_{1}\right)=i^{-1} e=i^{-1}$ and $l\left(\pi_{2}\right)=e^{-1} j=j$. It remains to find $l(\sigma)$.

If $e=f$, then, by Lemma $8, l(\sigma) \in\left\{e, e^{\prime}\right\}$. Suppose that $e \neq f$. We cannot get from $e$ to $f$ without passing $e_{o}$. Therefore, $\sigma$ is a concatenation of a loop $\sigma_{1}$ from $e$ to $e$ with all of its vertices in $G_{e}$, the labeled arrow $e \stackrel{b_{e}}{\longrightarrow} e_{o}$ (if $e \neq e_{o}$ ), a loop $\sigma_{2}$ from $e_{o}$ to $e_{o}$, the labeled arrow $e_{o} \stackrel{a_{f}}{\longrightarrow} f$ (if $f \neq e_{o}$ ), and a loop $\sigma_{3}$ from $f$ to $f$ with all of its vertices in $G_{f}$. By Lemma $6, l\left(\sigma_{1}\right)=e$ and $l\left(\sigma_{3}\right)=f$. By Lemma $8, l\left(\sigma_{2}\right)=e_{o}$. Therefore, $l(\sigma)=e b_{e} e_{o} a_{f} f=b_{e} a_{f}=q_{e, f}$. It follows that $l(\pi)=l\left(\pi_{1}\right) l(\sigma) l\left(\pi_{2}\right)=i^{-1} q_{e, f} j$.

Define $Q_{e}(s)=Q_{\tilde{\mu}}(s) \cap G_{e} \times G_{e}$ for all $s \in G_{e}$.

Lemma 10. $Q_{e}=\mu_{e}$ for all $e \in E(\mathcal{S})$.

Proof. Obviously, $\mu_{e}(s) \subset \mu(s) \subset \tilde{\mu}(s) \subset Q_{\tilde{\mu}}(s)$, and so $\mu_{e}(s) \subset Q_{e}(s)$. If $(i, j) \in$ $Q_{e}(s)$ for $i, j, s \in G_{e}$, then there exists a labeled path $\pi: i \rightarrow \cdots \rightarrow j$ in $\tilde{\mu}$ of length $s$. This path is a concatenation of a path $\pi_{1}$ from $i$ to $e$ with vertices in $G_{e}$, followed by a cycle $\pi_{2}$ from $e$ to $e$ with vertices in $\mathcal{G}(\mathcal{S})$, and a path $\pi_{3}$ from $e$ to $j$ with vertices in $G_{e}$. By Lemma $8, l\left(\pi_{2}\right) \in\left\{e, e^{\prime}\right\}$.

Suppose that $l\left(\pi_{2}\right)=e^{\prime}$. Let $l\left(\pi_{1}\right)=u$ and $l\left(\pi_{3}\right)=v$, where $u, v \in G_{e}$. Then $u e^{\prime} v=l\left(\pi_{1}\right) l\left(\pi_{2}\right) l\left(\pi_{3}\right)=l\left(\pi_{1} \pi_{2} \pi_{3}\right)=l(\pi)=s \in G_{e}$. If $x \in G_{e}$, let $x^{-1}$ be the inverse of $x$ in $G_{e}$. Then $e^{\prime}=e e^{\prime} e=u^{-1} u e^{\prime} v v^{-1}=u^{-1} s v^{-1} \in G_{e}$, and hence $e^{\prime}=e$, because $G_{e}$ has only one idempotent.

It follows that $l\left(\pi_{1} \pi_{3}\right)=l\left(\pi_{1}\right) l\left(\pi_{3}\right)=l\left(\pi_{1}\right) e\left(\pi_{3}\right)=l\left(\pi_{1}\right) l\left(\pi_{2}\right) l\left(\pi_{3}\right)=l\left(\pi_{1} \pi_{2} \pi_{3}\right)=$ $l(\pi)=s$, where $\pi_{1} \pi_{3}$ is a path from $i$ to $j$ with all of its vertices in $G_{e}$. Thus $(i, j) \in \mu_{e}(s)$, and hence $Q_{e}(s) \subset \mu_{e}(s)$.

Lemma 11. Every simple semigroup $\mathcal{S}$ possesses a transitive quasi-representation $Q$ such that the restriction $\left.Q\right|_{G_{e}}$ of $Q$ to each $G_{e}$ is injective.

Proof. If $\mathcal{G}(\mathcal{S})=\emptyset$, let $Q(s)=\{(0,0)\}$ for all $s \in S$. Obviously, $Q$ is a transitive representation of $\mathcal{S}$ by relations on a set $\{0\}$. Also, $Q$ is injective on all subgroups of $\mathcal{S}$ (because there are none). If $\mathcal{G}(\mathcal{S}) \neq \emptyset$, use Step III to construct the quasirepresentation $Q=Q_{\tilde{\mu}}$. We have seen that $Q$ is transitive. If $Q(s)=Q(t)$ for some $s, t \in G_{e}$ and $e \in E(\mathcal{S})$, then, by Lemma $10, \mu_{e}(s)=Q_{e}(s)=Q(s) \cap G_{e} \times G_{e}=$ 
$Q(t) \cap G_{e} \times G_{e}=Q_{e}(t)=\mu_{e}(t)$, and hence $s=t$, because the Cayley representation $\mu_{e}$ is injective. Thus $Q$ is injective on maximal subgroups of $\mathcal{S}$.

Step IV. Here we define a quasi-representation $Q_{\check{\nu}}$.

Let $\nu: S \rightarrow \mathcal{B}_{A}$ be a transitive quasi-representation of a simple semigroup $\mathcal{S}$ such that $\nu$ is injective on all subgroups of $\mathcal{S}$ and $A \neq \emptyset$, and let $X=\{(s, t) \in$ $S \times S \mid \nu(s)=\nu(t) \wedge s \neq t \wedge s \notin t s S s t\}$.

If $s \in t s S s t$ and $t \in$ stSts for some $s, t \in S$, then $s=t s x s t$ and $t=$ styts for suitable $x, y \in S$. Let $a=s x s t, b=t y t s, c=t s x s$, and $d=s t y t$. Then $s=t a=c t$ and $t=s b=d s$, so that $t=t a b$ and $s=c t=c t a b=s a b$. Analogously, $c d s=s$, and hence $c d t=t$. Therefore, $a b=s x \cdots=(c d s) x \cdots=(c d)(a b)=$ $(\cdots y t)(a b)=\cdots y(t a b)=\cdots y t=c d$. The element $e=a b=c d$ is idempotent, and hence $s, t \in G_{e}$. If $\nu(s)=\nu(t)$, then $s=t$, because $\nu$ is injective on $G_{e}$. Thus, if $\nu(s)=\nu(t)$ and $s \neq t$, then $(s, t) \in X$ or $(t, s) \in X$.

Since $A \neq \emptyset$ and $\nu$ is transitive, we can choose $w \in S$ and $i, j \in A$ such that $(i, j) \in \nu(w)$. Next choose $u_{s, t}, v_{s, t} \in S$ such that $w=u_{s, t} s t s v_{s, t}$.

For every pair $(s, t) \in X$ add four new vertices $a_{s, t}, b_{s, t}, c_{s, t}$ and $d_{s, t}$ to $A$. Let $\check{A}$ be the extended set of vertices. Also, for every $(s, t) \in X$ add to $\nu$ the following five labeled arrows: $i \stackrel{u_{s, t}}{\longrightarrow} a_{s, t} \stackrel{s}{\rightarrow} b_{s, t} \stackrel{t}{\rightarrow} c_{s, t} \stackrel{s}{\rightarrow} d_{s, t} \stackrel{v_{s, t}}{\longrightarrow} j$. Let $\check{\nu}$ denote the new labeled multi-graph $S \rightarrow \mathcal{B}_{\check{A}}$ and let $Q_{\check{\nu}}$ be the quasi-representation of $\mathcal{S}$ corresponding to the multigraph $\check{\nu}$. Clearly, $\check{\nu}$ is transitive, and hence $Q_{\check{\nu}}$ is transitive.

Lemma 12. Every simple semigroup admits a faithful transitive representation by binary relations.

Proof. Suppose that $Q_{\check{\nu}(s)}=Q_{\check{\nu}(t)}$ for some $s, t \in S$. If $s$ and $t$ belong to the same subgroup of $\mathcal{S}$, then $s=t$ because $\check{\nu}$ is injective on the subgroups. Suppose that $s \neq t$ and $s$ and $t$ do not belong to the same subgroup. Then $(s, t) \in X$ or $(t, s) \in X$. Without loss of generality, let $(s, t) \in X$. Then $\left(b_{s, t}, c_{s, t}\right) \in \check{\nu}(t) \subset Q_{\check{\nu}(t)}$, and hence $\left(b_{s, t}, c_{s, t}\right) \in Q_{\check{\nu}(s)}$. Thus there exists a labeled path $\pi$ of length $s$ in $\check{\nu}$ leading from $b_{s, t}$ to $c_{s, t}$. Since $s \neq t$, this path contains more than one arrow. However, the only path in $\check{\nu}$ leading from $b_{s, t}$ that has more than one arrow is $b_{s, t} \stackrel{t}{\rightarrow} c_{s, t} \stackrel{s}{\longrightarrow} d_{s, t} \longrightarrow \cdots$, and the only path leading to $c_{s, t}$ that is not a single arrow is $\cdots \rightarrow a_{s, t} \stackrel{s}{\rightarrow} b_{s, t} \stackrel{t}{\rightarrow} c_{s, t}$. Therefore, $s=l(\pi)=t s \ldots s t \in t s S s t$, which contradicts $(s, t) \in X$. Thus $s=t$.

Let $R_{\omega}$ be a free representation of $\mathcal{S}$ generated by $\check{\nu}$. It is transitive and faithful because it extends the transitive and injective $Q_{\check{\nu}}$.

Now we can complete the proof of Theorem A. If a semigroup $\mathcal{S}$ has no kernel, apply Lemma 5 . If $\mathcal{S}$ has a kernel $K$, then $K$ is a simple semigroup. Apply Lemma 12 and then Lemma 4.

\section{A REMARK-ESSAY ON "METRICS" IN MULTI-GRAPHS}

To prove Theorem A we used a "metric" on multi-graphs. This idea can be (and has been) applied to other situations, this is why we discuss it now in more detail. As we have already seen, if we want to consider "metrics" in multi-graphs, it is natural to assume that the length of a path should be the "sum" of lengths of its arrows in the order in which they are passed. Thus it is natural to assume that the set $S$, from which we take "lengths" of arrows, has a binary operation (following 
tradition, we call the result of its application a "product" rather than "sum"). Looking at the length of a three-arrow path $\cdot \stackrel{x}{\longrightarrow} \cdot \stackrel{y}{\longrightarrow} \cdot \stackrel{z}{\longrightarrow} \cdot$, we see that it is natural to assume that $(x y) z=x(y z)$ for all $x, y, z \in S$. Thus it is natural to use semigroups (or at least small categories) as sets of possible distances in multi-graphs.

If we want our "metrics" to satisfy other "natural" properties, we may have to impose specific conditions on the semigroup $\mathcal{S}$ of "distances." For example, it may be natural to ask what is the length of a trivial path with a single vertex and no arrows in it. Our "metric intuition" tells us this is "zero." Analyzing that intuition, we see that the length in this case should not "add anything" to the length of any path. Thus, if the length of an "empty" path $(i)$ with a single vertex $i$ is $a$ and $\pi$ is a path of length $s$ beginning at $i$, then the length of the concatenation of $(i)$ and $\pi$ should be the same as $l(\pi)$, that is, as $=s$. Concatenating $(i)$ with itself, we see that $a a=a$, that is, $a$ is an idempotent. Analogously, considering paths of length $t$ ending at $i$ and concatenating them with $(i)$, we see that $t a=t$. Thus, $a$ is an idempotent left identity or a right identity for certain elements of $\mathcal{S}$. If we prefer the length of $(i)$ not to depend on the choice of the vertex $i$, than this length must be the identity element of $\mathcal{S}$; that is, it is natural to assume that $\mathcal{S}$ is a monoid. If the length of $(i)$ depends on $i$, we obtain a semigroup with specific conditions (for example, each of its elements has left and right idempotent identities).

What about the "symmetry" of our "metric"? One obstacle is that the arrows in our multi-graphs are oriented and passed in the direction of their orientation in any path. It is possible to ask what might be the length of an arrow $i \stackrel{s}{\rightarrow} j$ if it is passed in the opposite direction, from $j$ to $i$. One possibility is to consider non-oriented arrows. Then, if we want our metric to be symmetric, we have to conclude that the length st of a path $i \stackrel{s}{-}{ }_{j} \stackrel{t}{-} k$ should coincide with the length of that path passed in the opposite direction, from $k$ to $i$, and hence $s t=t s$. Thus we have to assume that our "metric semigroup" $\mathcal{S}$ is commutative, and we may use a more intuitively appealing additive terminology for lengths of paths.

However, in certain situations (for example, for representations by binary relations) arrows of our multi-graphs are oriented and commutativity of $\mathcal{S}$ may not be a natural condition. Another approach is possible. Assume that if two arrows $i_{1} \rightarrow j_{1}$ and $i_{2} \rightarrow j_{2}$ have the same length $s$, then those arrows passed in the opposite direction also have the same length, say $s^{-1}$. In other words, assume that the length of an arrow $i \stackrel{s}{\rightarrow} j$ passed in the opposite direction depends on $s$ but not on the endpoints $i$ and $j$. Then $s \rightarrow s^{-1}$ is a unary operation in $\mathcal{S}$. If we reverse the orientation of an arrow $\stackrel{s}{\rightarrow}$ twice, its length $s$ will change as follows: $s \rightarrow s^{-1} \rightarrow\left(s^{-1}\right)^{-1}$. However, after two reversals we return to the original arrow, and hence it is natural to assume that $\left(s^{-1}\right)^{-1}=s$ for any $s \in S$.

If $\pi: \stackrel{s}{\rightarrow} \cdot \stackrel{t}{\rightarrow} \cdot$ is a path of length $s t$, it is natural to assume that its length in the opposite direction would be $(s t)^{-1}$. However, it is no less natural to assume that the length of $\pi$ in the opposite direction would be the product $t^{-1} s^{-1}$. Thus we obtain $(s t)^{-1}=t^{-1} s^{-1}$ for any $s, t \in S$.

An algebra of the form $\mathcal{S}=\left(S ; \cdot,^{-1}\right)$ is called an involuted semigroup if $(S ; \cdot)$ is a semigroup, ${ }^{-1}$ is a unary operation, and the identities $\left(x^{-1}\right)^{-1}=x$ and $(x y)^{-1}=$ $y^{-1} x^{-1}$ hold in $\mathcal{S}$. Thus, when we measure lengths of arrows in the direction opposite to their orientation, we may consider involuted semigroups.

If the distances in a multi-graph are measured by elements of an involuted semigroup, we may determine lengths of all sorts of "zigzags." For example, consider 
the configuration $i \stackrel{s}{\rightarrow} j \stackrel{t}{\leftarrow} k \stackrel{u}{\rightarrow} l$. This is not a path, but we can turn it into a path by reversing the orientation of $j \leftarrow k$. The length of that path would be $s t^{-1} u$.

Now suppose that we want to pass an arrow $i \stackrel{s}{\rightarrow} j$ from $i$ to $j$, then return to $i$, and then return back to $j$. The length of this "zigzag path" would be $s s^{-1} s$. Is this zigzag path "longer" than the original arrow $i \stackrel{s}{\rightarrow} j$ ? To compare lengths of different paths we need something like an order relation, we need a binary relation $s \prec t$, that means that $s$ is "shorter than or equal to" $t$. Obviously, this relation should be reflexive: $s \prec s$, and transitive: $s \prec t \wedge t \prec u \Rightarrow s \prec u$. It may be antisymmetric: if $s$ is shorter than $t$ and $t$ shorter than $s$, then $s=t$, but situations are conceivable in which $\prec$ does not have to be antisymmetric. Also, $\prec$ does not have to be linear: neither $s \prec t$ nor $t \prec s$ may hold for certain $s, t \in S$. So, if we want to compare distances, our semigroup $\mathcal{S}$ should be equipped with a (partial) order relation, or at least with a quasi-order relation. There may be other natural conditions too. For example, if $i \stackrel{s_{1}}{\longrightarrow} j$ is shorter than $i \stackrel{s_{2}}{\longrightarrow} j$ and $j \stackrel{t_{1}}{\longrightarrow} k$ is shorter than $j \stackrel{t_{2}}{\longrightarrow} k$, then it is natural to assume that $i \stackrel{s_{1}}{\longrightarrow} j \stackrel{t_{1}}{\longrightarrow} k$ is shorter than $i \stackrel{s_{2}}{\longrightarrow} j \stackrel{t_{2}}{\longrightarrow} k$. In other words, $s_{1} \prec s_{2} \wedge t_{1} \prec t_{2} \Rightarrow s_{1} t_{1} \prec s_{2} t_{2}$, that is, $\prec$ is stable (or compatible with multiplication): $(\prec)(\prec) \subset(\prec)$, where $\prec$ is considered as a subset of the semigroup $\mathcal{S} \times \mathcal{S}$. What can we say about $s^{-1}$ and $t^{-1}$ if $s \prec t$ ? We should not jump to the conclusion $t^{-1} \prec s^{-1}$, typical for a group situation, because ${ }^{-1}$ does not necessarily denote the "reciprocal" or "inverse." Indeed, if $i \stackrel{s}{\rightarrow} j$ is shorter than $i \stackrel{t}{\rightarrow} j$, we may conclude that $i \stackrel{s^{-1}}{\longleftarrow} j$ is shorter than $i \stackrel{t^{-1}}{\longleftarrow} j$, that is, $s \prec t \Rightarrow s^{-1} \prec t^{-1}$.

Also, we can interpret $l(\pi)$ for a path $\pi$ as the "work" done when we move along $\pi$. In particular, we may consider "conservative fields" in which lengths of cycles are "zero," or at least an idempotent element. For example, if the work $s$ is done when we move along an arrow $i \rightarrow j$, then $i \stackrel{s}{\rightarrow} j$ is the corresponding labeled arrow, and we can conclude that the work is "undone" when we move back from $j$ to $i$ along the same arrow. Thus, the length $s s^{-1}$ of the path $i \stackrel{s}{\rightarrow} j \leftarrow i$ is $e$, where $e$ is the identity element of $\mathcal{S}$ (or at least $e$ is a "local identity"). This approach leads us to groups and inverse semigroups.

On the other hand, geometric intuition may tell us that $s \prec s s^{-1} s$ for all $s \in S$ because the length of a "zigzag" $i \stackrel{s}{\rightarrow} j \stackrel{s}{\longleftarrow} i \stackrel{s}{\rightarrow} j$ should be longer than or equal to the length of $i \stackrel{s}{\rightarrow} j$. This is a particular case of the "triangle inequality," and we may look into what the triangle inequality means in our situation. This is a fruitful approach, for it led to a satisfactory solution of another longstanding semigroup-theoretic problem. An involuted semigroup $\mathcal{S}$ is called representable by binary relations if it is isomorphic to an involuted semigroup $\left(\Phi ; \circ,{ }^{-1}\right)$ of binary relations on a set. Here, if $\varphi \in \Phi$, then $\varphi^{-1}=\{(i, j) \mid(j, i) \in \varphi\}$. The problem of characterizing involuted semigroups representable by binary relations, first raised in 1953, was solved in [6] using an aproach quite analogous to that used in the proof of Theorem A. It turns out that the representability of an involuted semigroup $\mathcal{S}$ is equivalent to a certain "triangular inequality" property for the metric on labeled multi-graphs associated with $\mathcal{S}$. This property gives rise to a system of quasi-identities that characterize axiomatically the class of representable involuted semigroups.

Another application of certain ideas used in the proof of Theorem A yielded a proof of the following theorem (see [1]): for every (partially) ordered semigroup 
$\mathcal{S}=(S ; \cdot ; \leq)$ there exists an isomorphism $\mathrm{P}$ onto an inclusion-ordered semigroup of binary relations $(\Phi ; \circ ; \subset)$ such that, for any nonempty subset $T \subset S$ for which the greatest lower bound $a=\inf T$ exists in $\mathcal{S}, \mathrm{P}(a)=\bigcap\{\mathrm{P}(t) \mid t \in T\}$ (thus $\mathrm{P}$ is an infima-preserving isomorphism). In particular, the construction used in [1] leads to a representation $\mathrm{P}$ such that all the relations $\mathrm{P}(s)$ satisfy certain special conditions. It would be interesting to see if the construction from [1] could yield transitive representations by special types of relations.

Undoubtedly, metrics in labeled graphs can be used for solving other problems concerning semigroups of binary relations.

\section{Proof of Theorem B}

Recall that the set of idempotents of a semigroup is ordered by $\leq$ (defined in Step III). A completely simple semigroup is a simple semigroup with a minimal idempotent. Historically, finite simple semigroups (which are obviously completely simple) were the first class of semigroups, besides groups, for which a nontrivial structural theorem was found (see [7] and [8], or Appendix A in [2]).

In Step III we saw that $e^{\prime} \leq e$ for idempotents $e$ of a simple semigroup. In the completely simple case all idempotents are primitive (see [7], [8], or [3]), and hence $e^{\prime}=e$. Also, if $e \in E(\mathcal{S})$ and $e s \in G_{e}$ for an element $s$ of a completely simple semigroup $\mathcal{S}$, then $s \in G_{e}$ (for example, see Theorem 2.52(iii) of [2].) In this case Lemma 9 can be restated as follows.

Lemma 13. If $s \in S, i \in G_{e}$, and $j \in G_{f}$ for some $e, f \in E(\mathcal{S})$ in a completely simple semigroup $\mathcal{S}$, then the following are equivalent:

(i) $(i, j) \in Q_{\tilde{\mu}}(s)$;

(ii) $s=i^{-1} q_{e, f} j$;

(iii) $i s=q_{e, f} j$;

(iv) $j=q_{f, e} i s$.

Proof. The alternative lengths in Lemma 9 coincide because $e^{\prime}=e$ for all $e \in E(\mathcal{S})$. By Lemma $9,(i) \Leftrightarrow(i i)$. If (ii) holds, then $i s=i i^{-1} q_{e, f} j=e q_{e, f} j=q_{e, f} j$, which is (iii). Also, $q_{e, f} q_{f, k}=b_{e} a_{f} b_{f} a_{k}=b_{e} e_{o} a_{k}=b_{e} a_{k}=q_{e, k}$ for any $e, f, k \in E(\mathcal{S})$. In particular, $q_{f, e} q_{e, f}=q_{f, f}=f$. Thus (iii) implies $q_{f, e} i s=q_{f, e} q_{e, f} j=f j=j$, which is $(i v)$. If $(i v)$ is true, then $s=e s=i^{-1} e i s=i^{-1} q_{e, f} q_{f, e} i s=i^{-1} q_{e, f} j$, which is $(i i)$.

Lemma 14. For a completely simple semigroup, $Q_{\tilde{\mu}}$ is a faithful transitive representation by binary relations.

Proof. A completely simple semigroup is a union of its maximal subgroups. Let $H_{s}$ denote the maximal subgroup that contains $s \in S$ (thus $H_{e}=G_{e}$ for $e \in E(\mathcal{S})$ ). Then $H_{s} H_{t} \subset H_{s t}$ and $H_{s} H_{t} H_{s} \subset H_{s}$ for all $s, t \in S$ (see [2]).

Let $(i, j) \in Q_{\tilde{\mu}}(s t)$ for some $s, t \in S, i \in G_{e}$, and $j \in G_{f}$. By Lemma 13, $j=q_{f, e} i s t$. Let $g$ be the identity element of the subgroup $H_{t s}$. Define $k=q_{g, e} i s=$ $g q_{g, e} i s \in H_{t s} S H_{s} \subset H_{t} H_{s} S H_{s} \subset H_{t s}=G_{g}$. By Lemma 13, $(i, k) \in Q_{\tilde{\mu}}(s)$. Also, $k t=q_{g, e} i s t=q_{g, f} q_{f, e} i s t=q_{g, f} j$. By Lemma $13,(k, j) \in Q_{\tilde{\mu}}(t)$. It follows that $(i, j) \in Q_{\tilde{\mu}}(s) \circ Q_{\tilde{\mu}}(t)$, so that $Q_{\tilde{\mu}}(s t) \subset Q_{\tilde{\mu}}(s) \circ Q_{\tilde{\mu}}(t)$, and hence $Q_{\tilde{\mu}}(s t)=$ $Q_{\tilde{\mu}}(s) \circ Q_{\tilde{\mu}}(t)$ for all $s, t \in S$, because $Q_{\tilde{\mu}}$ is a transitive quasi-representation. Thus $Q_{\tilde{\mu}}$ is a transitive representation. 
Suppose that $Q_{\tilde{\mu}}(s)=Q_{\tilde{\mu}}(t)$ for some $s, t \in S$. If $s \in G_{e}$, then $s=e s=e q_{e, e} s$, and, by Lemma $13,(e, s) \in Q_{\tilde{\mu}}(s)$. Therefore, $(e, s) \in Q_{\tilde{\mu}}(t)$, and hence $t=e q_{e, e} s=$ $s$. Therefore, $Q_{\tilde{\mu}}$ is faithful.

Remark. The representation $Q_{\tilde{\mu}}$ for completely simple semigroups was defined (with a different notation) in [5]. We mention only a few of its remarkable properties. A representation $R: S \rightarrow \mathcal{B}_{A}$ is called simply transitive if, for any $(a, b) \in A \times A$, there exists exactly one $s$ in $\mathcal{S}$ such that $(a, b) \in R(s)$. As proved in [5], a semigroup $\mathcal{S}$ has a faithful simply transitive representation if and only if it is completely simple or completely 0 -simple (the latter case occurs when $\mathcal{S}$ has a zero represented by the empty binary relation). Thus completely simple semigroups are characterized by the fact of being isomorphic to simply transitive semigroups of nonempty binary relations. If $\mathrm{P}: \mathcal{S} \rightarrow \mathcal{B}_{A}$ is a faithful simply transitive representation of a completely simple semigroup, inflate it as follows. For a set $B$ and a mapping $\varphi: B \rightarrow A$ of $B$ onto $A$, define $\mathrm{P}_{\varphi}: S \rightarrow \mathcal{B}_{B}$ by the formula $(b, c) \in \mathrm{P}_{\varphi}(s) \Leftrightarrow(\varphi(b), \varphi(c)) \in \mathrm{P}(s)$ for all $b, c \in B$. Thus every pair $(i, j) \in A \times A$ is replaced by a "rectangle" $\varphi^{-1}(i) \times \varphi^{-1}(j) \subset B \times B$. It is not difficult to see that $\mathrm{P}_{\varphi}$ is a faithful simply transitive representation of $\mathcal{S}$. It turns out that every faithful simply transitive representation of $\mathcal{S}$ is an inflation of a representation (called canonical) that is not a nontrivial inflation of any representation. Any two canonical representations of $\mathcal{S}$ are similar. Here representations $R_{1}: S \rightarrow \mathcal{B}_{A_{1}}$ and $R_{2}: S \rightarrow \mathcal{B}_{A_{2}}$ are called similar if there exists a bijection $\theta: A_{1} \rightarrow A_{2}$ such that $(a, b) \in R_{1}(s) \Leftrightarrow(\theta(a), \theta(b)) \in$ $R_{2}(s)$ for all $s \in S$ and $a, b \in A_{1}$. It is easy to see that the representation $Q_{\tilde{\mu}}$ from Lemma 14 is simply transitive. It turns out that it is exactly the canonical representation of $\mathcal{S}$ introduced in [5].

Let $\mathcal{S}$ be a semigroup with a completely simple kernel $K$. Extend the canonical representation $Q_{\tilde{\mu}}$ of $K$ to a mapping $R: S \rightarrow \mathcal{B}_{K}$ by defining $(i, j) \in R(s) \Leftrightarrow i s=$ $q_{e, f} j$ for every $s \in S, i \in G_{e}$, and $j \in G_{f}$, where $e, f \in E(K)$.

Lemma 15. $R$ is a transitive representation of $\mathcal{S}$. It is injective on $K$, and $R(s) \neq$ $\emptyset$ for all $s \in S$.

Proof. Let $(i, j) \in R(s)$ and $(j, k) \in R(t)$ for $i \in G_{e}, j \in G_{f}$, and $k \in G_{g}$, where $G_{e}$, $G_{f}$, and $G_{g}$ are certain maximal subgroups of $K$. Then $i s=q_{e, f} j$ and $j t=q_{f, g} k$ in $K$. It follows that $i s t=q_{e, f} j t=q_{e, f} q_{f, g} k=q_{e, g} k$, and hence $(i, k) \in R(s t)$.

Suppose that $(i, k) \in R(s t)$ for some $i \in G_{e}$ and $k \in G_{g}$. Then $k g=k$ and $i s t=q_{e, g} k$, whence $i s t=q_{e, g} k=q_{e, g} k g=i s t g$. Also, $i s, t g \in K$, and, by Lemma $13,(i, k) \in Q_{\tilde{\mu}}(i s \cdot t g)=Q_{\tilde{\mu}}(i s) \circ Q_{\tilde{\mu}}(t g)$. Thus $(i, j) \in Q_{\tilde{\mu}}(i s)$ and $(j, k) \in Q_{\tilde{\mu}}(t g)$ for some $j \in G_{f}$, where $f$ is a suitable idempotent of $K$. By Lemma 13 , is = $q_{e, f} j$ and $j t g=q_{f, g} k$. It follows from $j f=j$ that $i s f=q_{e, f} j g=q_{e, f} j=i s$. Principal left ideals of a completely simple semigroup are minimal (see [2]). Thus $f t \in K f t=K i s \cdot f t=K i s t=K i s t g \subset K t g$, and hence $f t=x t g$ for some $x \in K$. It follows that $j t=j f t=j x t g=j x t g g=j t g=q_{f, g} t$. Therefore, $(i, j) \in R(s)$ and $(j, k) \in R(t)$, which implies $(i, k) \in R(s) \circ R(t)$. Thus $R(s t)=R(s) \circ R(t)$, so that $R$ is a representation of $\mathcal{S}$. By the definition of $R$ and Lemma 13, $R$ coincides with $Q_{\tilde{\mu}}$ on $K$, and, by Lemma $14, R$ is injective on $K$. Also, $R$ is transitive because $Q_{\tilde{\mu}}$ is transitive.

It is easy to see that $\left(e_{o}, e_{o}\right) \in Q_{\tilde{\mu}}\left(e_{o}\right)$. Since $K$ is simple, it follows that $e_{o}=x t y$ for each $t \in K$ and some $x, y \in K$. Thus $Q_{\tilde{\mu}}\left(e_{o}\right)=Q_{\tilde{\mu}}(x) \circ Q_{\tilde{\mu}}(t) \circ Q_{\tilde{\mu}}(y)$, and 
hence $Q_{\tilde{\mu}}(t) \neq \emptyset$. Finally, $k s \in K$ for every $k \in K$ and $s \in S$. It follows that $R(k) \circ R(s)=R(k s)=Q_{\tilde{\mu}}(k s) \neq \emptyset$, and hence $R(s) \neq \emptyset$.

Lemma 16. Every finite semigroup with zero admits a faithful transitive representation by nonempty binary relations on a finite set.

Proof. In the proof of Lemma 4 we have already constructed a faithful transitive representation for a semigroup $\mathcal{S}$ with zero 0 . Here we produce another representation. Let $M$ be the set of all subsets of $\mathcal{S}^{1}$ that contain 0 , where $\mathcal{S}^{1}$ is the semigroup obtained from $\mathcal{S}$ by adjoining an identity 1 (see [2]).

Define $Z: S \rightarrow \mathcal{B}_{M}$ as follows: $(\alpha, \beta) \in Z(s) \Leftrightarrow \alpha s \subset \beta$ for any $\alpha, \beta \in M$ and $s \in S$. If $(\alpha, \gamma) \in Z(s) \circ Z(t)$, then $(\alpha, \beta) \in Z(s)$ and $(\beta, \gamma) \in Z(t)$ for some $\beta \in M$, so that $\alpha s \subset \beta$ and $\beta t \subset \gamma$. It follows that $\alpha s t \subset \beta t \subset \gamma$, and hence $(\alpha, \gamma) \in Z(s t)$. Conversely, suppose that $(\alpha, \gamma) \in Z(s t)$, that is, $\alpha s t \subset \gamma$. Let $\beta=\alpha s$. Then $0=0 s \in \alpha s=\beta$, so that $\beta \in M$. Obviously, $(\alpha, \beta) \in Z(s)$ and $(\beta, \gamma) \in Z(t)$. It follows that $Z(s) \circ Z(t)=Z(s t)$ for all $s, t \in S$. Clearly, $(\alpha, \beta) \in Z(0)$ for all $\alpha, \beta \in M$. Thus $Z$ is a transitive representation of $\mathcal{S}$ by binary relations. If $s \in S$, then $\{0,1\} s=\{0, s\}$, and so $(\{0,1\},\{0, s\}) \in Z(s)$. It follows that $Z(s) \neq \emptyset$.

If $Z(s)=Z(t)$ for $s, t \in S$, then $(\{0,1\},\{0, s\}) \in Z(t)$, so that $t \in\{0,1\} t \subset$ $\{0, s\}$. Interchanging the roles of $s$ and $t$, we obtain $s \in\{0, t\}$. It follows that $s=t$, and hence the representation $Z$ is faithful. It remains to observe that, if $\mathcal{S}$ is finite, then $M$ is finite.

Remark. The representation $Z$ considered in the proof of Lemma 16 first appeared in [4].

Now we can complete the proof of Theorem B. A finite semigroup $\mathcal{S}$ has a completely simple kernel $K$. By Lemma $15, R$ is a transitive representation of $\mathcal{S}$ by relations on $K$.

Consider the Rees factor semigroup $T=\mathcal{S} / K$. Its elements are $(S \backslash K) \cup\{0\}$, where 0 is the zero and the multiplication in $S \backslash K$ is defined in the same way as in $\mathcal{S}$, except that whenever the product belongs to $K$, it is 0 (see [2]). Then $T$ is a finite semigroup with zero and a homomorphic image of $\mathcal{S}$ under an obvious homomorphism $\varphi: S \rightarrow T$. By Lemma 16, $T$ has a faithful transitive representation $Z$ by binary relations on a finite set $M$. Combining $\varphi$ and $Z$, we obtain a transitive representation $\zeta=\varphi \circ Z$ of $\mathcal{S}$.

If $R_{i}: S \rightarrow \mathcal{B}_{A_{i}}$ are representations of $\mathcal{S}$ for $i=1,2$, construct a representation $R_{1} \square R_{2}: S \rightarrow \mathcal{B}_{A_{1} \times A_{2}}$ as follows: $\left(\left(a_{1}, a_{2}\right),\left(b_{1}, b_{2}\right)\right) \in R_{1} \square R_{2}(s) \Leftrightarrow\left(a_{i}, b_{i}\right) \in R_{i}(s)$ for $i=1,2, a_{i}, b_{i} \in A_{i}$, and $s \in S$.

Let $\rho=R \square \zeta$. It is a representation of $\mathcal{S}$ by binary relations on a set $K \times M$. Let $(i, \alpha),(j, \beta) \in K \times M$. There exists $s \in K$ such that $(i, j) \in Q_{\tilde{\mu}}(s)=R(s)$. Also, $\varphi(s)=0 \in T$, and $Z(0)=M \times M$ (see our proof of Lemma 16). Thus $(\alpha, \beta) \in \zeta(s)$, and hence $((i, \alpha),(j, \beta)) \in \rho(s)$. It follows that $\rho$ is a transitive representation of $\mathcal{S}$.

Suppose that $\rho(s)=\rho(t)$ for some $s, t \in S$; then $R(s) \times \zeta(s)=R(t) \times \zeta(t)$ and, since $R(s)$ and $\zeta(s)$ are nonempty for all $s \in S$, we obtain $R(s)=R(t)$ and $\zeta(s)=\zeta(t)$. If $s \notin K$ or $t \notin K$, then $\zeta(s)=\zeta(t)$ implies $s=t$. If $s, t \in K$, then $R(s)=R(t)$ implies $s=t$, by Lemma 15 . Thus $\rho$ is faithful.

Since $\mathcal{S}$ is finite, both $K$ and $M$ are finite, and hence $K \times M$ is finite. 


\section{REFERENCES}

[1] D. A. Bredihin and B. M. Schein, Representations of ordered semigroups and lattices by binary relations, Colloquium Mathematicum 39 (1978), 1-12. MR 80c:20093

[2] A. H. Clifford and G. B. Preston, The Algebraic Theory of Semigroups. Vol. I, American Mathematical Society, Providence, R. I., 1961. MR 24:A2627

[3] D. Rees, Note on semi-groups, Proceedings of the Cambridge Philosophical Society 37 (1941), 434-435. MR 3:199b

[4] Б. М. Шайн, Представление полугрупп при помощи бинарных отношений, Математический Сборник 60 (1963), 293-303 [B. M. Schein, Representation of semigroups by means of binary relations, Matematicheskiı Sbornik 60 (1963), 292-303]. MR 27:3721

[5] B. M. Schein, A new representation theorem on completely [0]-simple semigroups, Semigroup Forum 4 (1972), 312-320. MR 47:6923

[6] B. M. Schein, Representation of involuted semigroups by binary relations, Fundamenta Mathematicæ 82 (1974), 121-141. MR 50:2381

[7] А. К. Сушкевич, Теория Действия, как Общая Теория Групп, Воронеж, 1922 [A. K. Suschkewitsch, "Theory of Operation as the General Theory of Groups," Voronezh, 1922].

[8] A. K. Suschkewitsch, Über die endlichen Gruppen ohne das Gesetz der eindeutigen Umkehrbarkeit, Mathematische Annalen 99 (1928), 30-50.

[9] К. А. Зарецкий, Представление упорядоченных полугрупп бинарными отношениями, Известия Высших Учебных Заведений, Математика 1959, nо. 6(13), 48-50

[K. A. Zaretskiŭ, Representation of ordered semigroups by binary relations, Izvestiya Vysshikh Uchebnykh Zavedeniı̌, Matematika 1959, no. 6(13), 48-50]. MR 24:A2630

Department of Mathematics, Vanderbilt University, 1326 Stevenson Center, Nashville, Tennessee 37240

E-mail address: mckenzie@math.vanderbilt.edu

Department of Mathematical Sciences, University of Arkansas, Fayetteville, ARKAnsas 72701

E-mail address: bschein@uafsysb.uark.edu 